\title{
Rehabilitación vestibular: perspectivas de implementación
}

\author{
Vestibular rehabilitation: prospects for implementation
}

\section{Sr. Editor:}

La disfunción vestibular produce inestabilidad postural, distorsión visual al movimiento de la cabeza y quejas de vértigo y desequilibrio, cuyas causas pueden ser lesiones periféricas (del laberinto o del nervio vestibular) o de las vías vestibulares centrales $(1,2)$.

La prevalencia de disfunción vestibular varía entre $29,3 \%$ y $35,4 \%(3,4)$. En relación a las atenciones de los trastornos del equilibrio y vértigo en urgencias hospitalarias, en EEUU fue de 3,3\% del total de urgencias (5).

Desde el punto de vista de la economía de la salud, en un estudio en el que participaron varios países,se evaluó el uso de recursos sanitarios en un periodo de tres meses. Los pacientes graves hicieron uso de 5,4 días en el hospital, el 93\% perdió días laborales, y el 22\% abandonó su trabajo (6). En otro estudio en Alemania, el 40,6\% informaron baja por enfermedad, el $18,5 \%$ evitó salir de la casa, y la interrupción de las actividades diarias se presentó en el 40,3\% de los casos (4).

La rehabilitación vestibular, considerada como parte deltratamiento integral de la disfunción vestibular, es un enfoque basado en ejercicios de estabilización de la mirada, de habituación, de equilibrio y de marcha (1); estos contribuyen a una mejora significativa del cuadro clínico y de la calidad de vida en los aspectos físicos, emocionales y funcionales (7).

Dada la alta prevalencia de disfunción vestibular, que genera carga social, sobre uso de recursos sanitarios y días laborales perdidos, asociado a la falta de oferta de la prestación de servicios de salud en rehabilitación vestibular y su eficacia a bajo costo, justifica la implementación de un programa oficial en el sector público.

\section{Raquel Tapia-Egoavil 1,a, Yuly Polo-Espinoza ${ }^{1, b}$, Janet Jara-López ${ }^{\text {1b }}$, Lizbeth Ramírez-García ${ }^{1, b}$}

\section{Correspondencia:}

Raquel Tapia

Correo electrónico: raqueltapiaego@gmail.com

\section{REFERENCIAS BIBLIOGRÁFICAS}

1. Hall CD, Herdman SJ, Whitney SL, et al. Vestibular rehabilitation for peripheral vestibular hypofunction: An evidence-based clinical practice guideline from the American Physical Therapy Association Neurologysection.JNeurol PhysTher.2016;40(2):12455. doi: 10.1097/NPT.0000000000000120

2. Kasper D, Fauci A, Hauser S, Longo D, Jameson L, Loscalzo J. Harrison Principios de Medicina Interna. New York: McGraw-Hill Interamericana Editores; 2016.

3. Agrawal Y,Carey J, Della Ch, Schubert M, Minor L. Disorders of balance and vestibular function in US Adults. Arch Intern Med. 2009;169(10):938-944.

4. Neuhauser HK, Radtke A, vonBrevern M, Lezius F, FeldmannM,LempertT.Burdenofdizzinessand vértigo inthecommunity.ArchInternMed.2008;168(19):211824. doi: 10.1001/archinte.168.19.2118

5. Newman-Toker DE, Hsieh YH, Camargo CA, Pelletier AJ, Butchy GT, Edlow JA. Spectrum of DizzinessVisitsto US Emergency Departments:

Departamento de Investigación, Docencia y Rehabilitación Integral en Amputados, Quemados y Trastornos Posturales, Instituto Nacional de Rehabilitación Dra. Adriana Rebaza Flores Amistad Perú - Japón. Chorrillos, Perú.

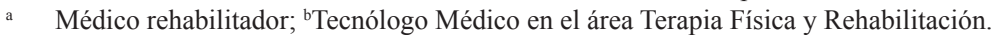


Cross-sectional analysis from a nationally representative sample. Mayo Clin Proc. 2008;83(7):765-75. doi: 10.4065/83.7.765

6. Benecke H, Agus S, Kuessner D, Goodall G, Strupp M. The Burden and Impact of Vertigo: Findings from the REVERT Patient Registry. Front Neurol. 2013; 4:136. doi: 10.3389/fneur.2013.0013
7. Soares S, Gonçalves M, Teixeira C, Romualdo $\mathrm{P}$, Santos J. Influência da reabilitação vestibular naqualidade de vida de individuos labirintopatas. Rev CEFAC.2014;16(3):732-738.Doi: http://dx.doi. org/10.1590/1982-0216201418211

Recibido: 03/03/2018 